\title{
An Efficient Explicit-time Description Method for Timed Model Checking
}

\author{
Hao Wang and Wendy MacCaull \\ Centre for Logic and Information \\ St. Francis Xavier University \\ Antigonish, Canada \\ $\{$ hwang, wmaccaul\}@stfx.ca
}

\begin{abstract}
Timed model checking, the method to formally verify real-time systems, is attracting increasing attention from both the model checking community and the real-time community. Explicit-time description methods verify real-time systems using general model constructs found in standard un-timed model checkers. Lamport proposed an explicit-time description method [17] using a clock-ticking process (Tick) to simulate the passage of time together with a group of global variables to model time requirements. Two methods, the Sync-based Explicit-time Description Method using rendezvous synchronization steps and the Semaphore-based Explicit-time Description Method using only one global variable were proposed [27, 26]; they both achieve better modularity than Lamport's method in modeling the real-time systems. In contrast to timed automata based model checkers like UPPAAL [7], explicit-time description methods can access and store the current time instant for future calculations necessary for many real-time systems, especially those with pre-emptive scheduling. However, the Tick process in the above three methods increments the time by one unit in each tick; the state spaces therefore grow relatively fast as the time parameters increase, a problem when the system's time period is relatively long. In this paper, we propose a more efficient method which enables the Tick process to leap multiple time units in one tick. Preliminary experimental results in a high performance computing environment show that this new method significantly reduces the state space and improves both the time and memory efficiency.
\end{abstract}

\section{Introduction}

Model checking is an automatic analysis method which explores all possible states of a modeled system to verify whether the system satisfies a formally specified property. It was popularized in industrial applications, e.g., for computer hardware and software, and has great potential for modeling complex and distributed business processes. Timed model checking, the method to formally verify real-time systems, is attracting increasing attention from both the model checking community and the real-time community. However, standard model checkers like SPIN [15] and SMV [19] can generally only represent and verify the qualitative relations between events, which constrains their use for real-time systems. Quantified time notions, including time instant and duration, must be taken into account for timed model checking. For example in a safety critical application such as in an emergency department, after an emergency case arrives at the hospital, standard model checking can only verify whether "the patient receives a certain treatment", but to save the patient's life, it should be verified whether "the patient receives a certain treatment within 1 hour".

Many formalisms with time extensions have been presented as the basis for timed model checkers. Two popular ones are: (1) timed automata [4], which is an extension of finite-state automata with a set of clock variables to keep track of time; (2) time Petri Nets [20], which is an extension of the Petri Nets with timing constraints on the firings of transitions. Various translation methods have been presented between

L. Brim and J. van de Pol (Eds.): 8th International Workshop on Parallel and Distributed Methods in verifiCation 2009 (PDMC'09) EPTCS 14, 2009, pp. 77-91 doi 10.4204/EPTCS.14.6 (c) H. Wang \& W. MacCaull

This work is licensed under the Creative Commons Attribution License. 
time Petri Nets to timed automata [22] in order to apply time-automata-based methods to time Petri Nets. UPPAAL [7] and KRONOS [28] are two well-known timed automata based model checkers; they have been successfully applied to various real-time controllers and communication protocols. Conventional temporal logics like Linear Temporal Logic (LTL) or Computation Tree Logic (CTL) must be extended [5] to handle the specification of properties of timed automata. In order to handle continuous-time semantics, specialized data structures are needed to represent real clock variables, e.g. Difference Bounded Matrices [12] (employed by UPPAAL and KRONOS).

The foundation for the decidability results in timed automata is based on the notion of region equivalence over the clock assignment [8]. Models in a timed automata based model checker can not represent at which time instant a transition is executed within a time region; such model checkers can only deal with a specification involving a time region or a pre-specified time instant and cannot store the exact time instant when the transition is executed. However, many real-time systems, especially those with pre-emptive scheduling, need this information for succeeding calculations. For example, triage is widely practiced in medical procedures; the caregiver $C$ may be administering some required but non-critical treatment on patient $A$ when another patient $B$ presents with a critical situation, such as a cardiac arrest. $C$ then must move to the higher priority task of treating $B$, but it is necessary to store the elapsed time of $A$ 's treatment to determine how much time is still needed or else the treatment must be restarted. The stop-watch automata [3], an extension of timed automata, is proposed to tackle this; unfortunately as Krcál and Yi discussed in [16], since the reachability problem for this class of automata is undecidable, there is no guarantee for termination in the general case.

Lamport [17] advocated explicit-time description methods using general model constructs, e.g., global integer variables or synchronization between processes commonly found in standard un-timed model checkers, to realize timed model checking. He presented an explicit-time description method, which we refer to as LEDM, using a clock-ticking process (Tick) to simulate the passage of time, and a pair of global variables to store the time lower and upper bounds for each modeled system process. The method has been implemented with popular model checkers SPIN (sequential) and SMV. We presented two methods, (1) the Sync-based Explicit-time Description Method (SEDM) [27] using rendezvous synchronization steps between the Tick and each of the system processes; and (2) the Semaphore-based Explicit-time Description Method (SMEDM) [26] using only one global semaphore variable. Both these methods enable the time lower and upper bounds to be defined locally in system processes so that they provide better modularity in system modeling and facilitate the use of more complex timing constraints. Our experiments [26, 27] showed that the time and memory efficiencies of these two methods are comparable to that of LEDM.

The explicit-time description methods have three advantages over timed-automata-based model checkers: (1) they do not need specialized languages or tools for time description so they can be applied in standard un-timed model checkers. Recently, Van den Berg et al. [9] successfully applied LEDM to verify the safety of railway interlockings for one of Australia's largest railway companies; (2) they enable the accessing and storing of the current time [26], a useful feature for pre-emptive scheduling problems; and (3) they enable the usage of large-scale distributed model checkers, e.g., DIVINE, for timed model checking.

Orthogonally, model checking has been studied in parallel and distributed computing platforms. Because real world models often come with gigantic state spaces which can not fit into the memory of a standard computer, inevitably a portion of the state space needs to be accessed from the secondary storage and the model checking algorithm becomes very slow [10]. This problem is known as state explosion. Large-scale analysis is needed in many practical cases. Distributed model checkers exploit the power of distributed computing facilities so that much larger memory is available to accommodate the state space 
of the system model; parallel processing of the states can, moreover, reduce the verification time. Our experiments [27] compared the time efficiency between the sequential SPIN and DIVINE [2], a wellknown distributed model checker. When using the same explicit-time description method, DiVINE can verify much larger models and finish the verification for models of the same size in significantly less time than SPIN.

In this paper, we present a new explicit-time description method called Efficient Explicit-time Description Method (EEDM). We found that the former three methods (LEDM, SEDM and SMEDM) suffer from one common problem: as the Tick process increments the time by one unit in each tick, the state space grows relatively fast as the time parameters increase. E.g., in our experiment [26] using LEDM, the number of states doubles as time bounds grow from 12 to 14. In the new EEDM, the Tick can increment the time in two modes: the standard mode and the leaping mode. When it is necessary to store the current time to allow access for future calculations, it ticks in the standard mode; otherwise, it ticks in the leaping mode. For each system process, we define one global variable indicating whether the process needs to store and access the current time, allowing the Tick process to switch between the standard mode and the leaping mode. For the experiments, we continue using DIVINE (the method is also applicable to other standard model checkers); the results show that: in the leaping mode, the number of states can be reduced significantly, so it is much less affected by the increase of time parameters; in the standard mode, the time and memory efficiencies are comparable with the former methods.

The remainder of the paper is organized as follows. Section 2 gives background information with respect to the DIVINE model checker. The new explicit-time description method implemented in DiVINE is presented in Section 3, for comparison, LEDM is also briefly described in the same section. Section 4 describes our experiments and the results. Section 5 concludes the paper.

\section{Preliminaries}

Section 2.1 is adapted from [25]; the syntax outlined in Section 2.2, while incomplete, is meant for the presentation of the time-explicit description methods; the complete description can be found in [2].

\subsection{Distributed Model Checking Algorithms in DIVINE}

DIVINE is an explicit-state LTL model checker based on the automata-based procedure by Vardi and Wolper [24]. The property to be specified is described by an LTL formula. In LTL model checking, all efficient sequential algorithms are based on the postorder exploration as computed by a depth-first search (DFS) of the state space. However, computing DFS postorder is P-complete [23], so no benefit in terms of either time or space will result from parallelization of this type of algorithm.

Two algorithms, OWCTY and MAP [6], are introduced in DIVINE. The sequential complexity of each is worse than that of the DFS-based algorithms but both can be efficiently implemented in parallel. OWCTY, or One Way to Catch Them Young, is based on the fact that a directed graph can be topologically sorted if and only if it is acyclic. The algorithm applies a standard linear topological sort algorithm to the graph. Failure in the sorting means the graph contains a cycle. Accepting cycles are detected with multiple rounds of the sorting. MAP, or Maximal Accepting Predecessors, is based on the fact that each accepting vertex in an accepting cycle is its own predecessor. To improve memory efficiency, the algorithm only stores a single representative accepting predecessor for each vertex by choosing the maximal one in a linear ordering of vertices.

These two algorithms are preferable in different cases. If the property of a model is expected to hold, 
and the state space can fit completely into (distributed) memory, OWCTY is preferable as it is three times faster than MAP to explore the whole state space. On the other hand, MAP can generally find a counterexample (if it exists) more quickly as it works on-the-fly.

\subsection{DIVINE Modeling Language}

DVE is the modeling language of DIVINE. Like in Promela (the modeling language of SPIN), a model described in DVE consists of processes, message channels and variables. Each process, identified by a unique name procid, consists of lists of local variable declarations and state declarations, the initial state declaration and a list of transitions.

A transition transfers the process state from stateid 1 to stateid $_{2}$. The transition may contain a guard (which decides whether the transition can be executed), a synchronization (which communicates data with another process) and an effect (which assigns new values to local or global variables). So we have

Transition : := stateid $_{1} \rightarrow$ stateid $_{2}\{$ Guard Sync Effect $\}$

The Guard contains the keyword guard followed by a boolean expression and the Effect contains the keyword effect followed by a list of assignments. The Sync follows the denotation for communication in CSP, '!' for the sender and '?' for the receiver. The synchronization can be either asynchronous or rendezvous. Value(s) is transferred in the channel identified by chanid. So we have

$$
\text { Sync : := sync chanid ! SyncValue | chanid ? SyncValue ; }
$$

A property process is automatically generated for the corresponding property written as an LTL formula. Modeled system processes and the property process progress synchronously, so the latter can observe the system's behavior step by step and catch errors.

\section{Explicit-Time Description Methods}

With explicit-time description methods, the passage of time and timed quantified values can be expressed in un-timed languages and properties to be specified can be expressed in conventional temporal logics. This section describes Lamport's LEDM before detailing our new EEDM. At the end of this section, we study a small pre-emptive example with respect to explicit-time description methods.

\subsection{The Lamport Explicit-time Description Method}

In LEDM, current time is represented with a global variable now that is incremented by an added Tick process. As we mentioned earlier, standard model checkers can only deal with integer variables, and a real-time system can only be modeled in discrete-time using an explicit-time description. So the Tick process increments now by 1 . Note that in explicit-time description methods for standard model checkers, the real-valued time variables must be replaced by integer-valued ones. Therefore, these methods in general do not preserve the continuous-time semantics; otherwise an inherently infinite-state specification will be produced and the verification will be undecidable. However, they are sound for a commonly used class of real-time systems and their properties [14].

Placing lower-bound and upper-bound timing constraints on transitions in processes is the common way to model real-time systems. Figure 1 shows a simple example of only two transitions: transition 


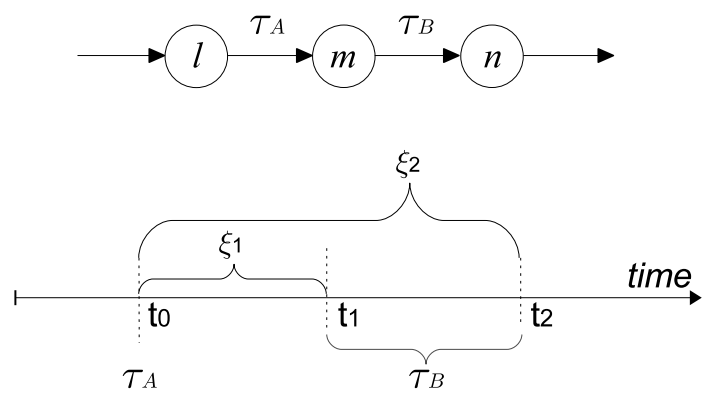

Figure 1: States and Timeline of process $P_{i}$

$\tau_{A}:$ stateid $_{l} \rightarrow$ stateid $_{m}$ is followed by the transition $\tau_{B}$ : stateid $_{m} \rightarrow$ stateid $_{n}$. An upper-bound timing constraint on when transition $\tau_{B}$ must occur is expressed by a guard on the transition in the Tick process so as to prevent an increase in time from violating the constraint. A lower-bound constraint on when transition $\tau_{B}$ may occur is expressed by a guard on $\tau_{B}$ so it cannot be executed earlier than it should be. Each system process $P_{i}$ has a pair of count-down timers denoted as global variables ubtimer $_{i}$ and lbtimer $_{i}$ for the timing constraints on its transitions. A large enough integer constant, denoted as INFINITY, is defined. All upper bound timers are initialized to INFINITY and all lower bound timers are initialized to zero. Upper bound timers with the value of INFINITY are not active and the Tick process will not decrement them. For transition $\tau_{B}$, the timers will be set to the correct values by $\tau_{A}$ : stateid ${ }_{l} \rightarrow$ stateid $_{m}$. As now is incremented by 1 , each non-INFINITY ubtimer and non-zero lbtimer is decremented by 1 .

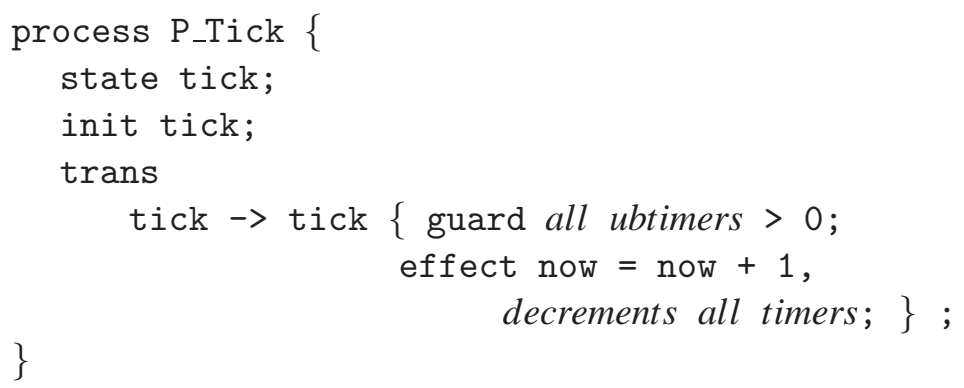

Figure 2: Tick process in DVE for LEDM

In Figure 1 initially, (ubtimer ${ }_{i}$, lbtimer $_{i}$ ) is set to (INFINITY,0). Transition $\tau_{A}$ is executed at time instant $t_{0}$, and $\left(\right.$ ubtimer $_{i}$, lbtimer $\left._{i}\right)$ is set to $\left(\xi_{2}, \xi_{1}\right)$. After $\xi_{1}$ time units, i.e., at time instant $t_{1}$ when (ubtimer ${ }_{i}$, lbtimer $\left._{i}\right)$ is equal to $\left(\xi_{2}-\xi_{1}, 0\right)$, transition $\tau_{B}$ is enabled. Both timers will be reset or set to new time bounds after the execution of $\tau_{B}$. If transition $\tau_{B}$ is still not executed when the time reaches $t_{2}$ and ubtimer $_{i}$ is equal to 0 , the transition in the Tick process is disabled. This forces transition $\tau_{B}$ (it is the only transition possible at this time) to set the ubtimer $_{i}$; then the Tick process can start again. In this way, the time upper-bound constraint is realized. The Tick process and the system process $P_{i}$ in DVE are described in Figure 2 and Figure 3 .

We observe that the value of now is limited by the size of type integer and careless incrementing can cause overflow error. This can be avoided by incrementing now using modular arithmetic, i.e., setting 


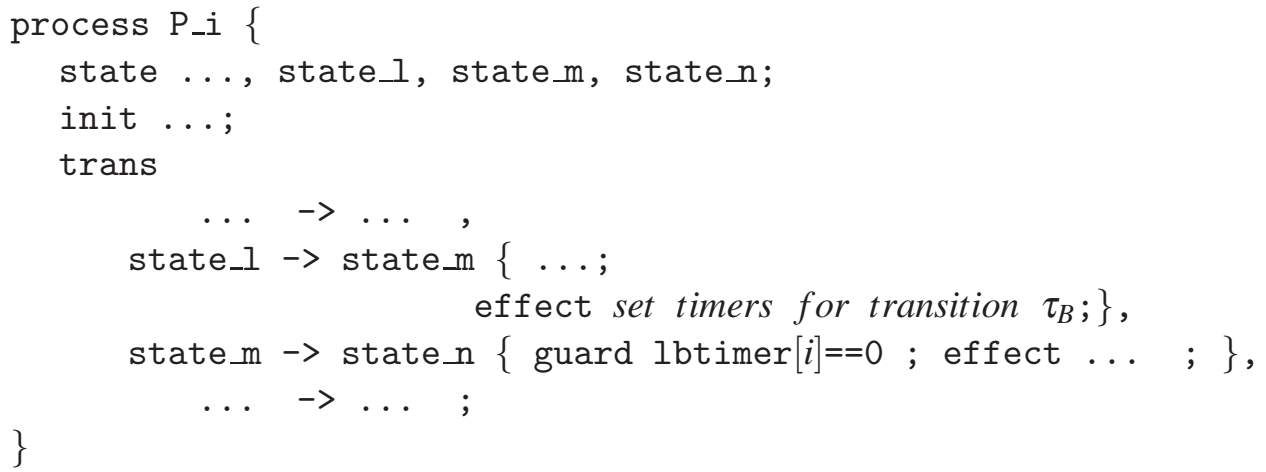

Figure 3: System process $P_{i}$ in DVE for LEDM

now $=($ now +1$)$ mod MAXIMAL (MAXIMAL is the maximal integer value supported by the model checker). The value limit can also be increased by linking several integers, i.e., every time $\left(i n t_{1}+1\right)$ mod MAXIMAL becomes zero again, int 2 increments by 1 , and so on. Note that the variable now is only incremented in the Tick process and does not appear in any other process. So for general system models in which time lower and upper bounds suffice, the variable now should be removed.

\subsection{The New Efficient Explicit-Time Description Method}

This section is organized as follows. First, we describe the leaping mode and the standard mode of the new EEDM in section 3.2.1 and 3.2.2 respectively. Second, we present some discussions (clarifications) of issues on EDMs and EEDM in section 3.2.3. Finally, a pre-emptive scheduling modeling example using EEDM is described in section 3.2.4.

\subsubsection{Leaping Ticks}

All aforementioned explicit-time description methods (LEDM, SEDM and SMEDM) increase now by 1 each tick. On the other hand, consider Figure 4 we observe that when the system contains only one process, $P_{i}$, after $t_{0}, \tau_{B}$ cannot be executed until time reaches $t_{2}$. Therefore, the ticks between $t_{0}$ and $t_{1}$ serve no purpose; optimally, the Tick process should directly "leap" to $t_{2}$. Similarly, $\tau_{B}$ is enabled between $t_{2}$ and $t_{4}$, so either $\tau_{B}$ is executed before $t_{4}$ or time reaches $t_{4}$ and $\tau_{B}$ 's execution is forced; therefore, the Tick process can leap to $t_{4}$ from $t_{2}$. When we include $P_{j}$, after $t_{0}$, the Tick should first leap to $t_{1}$ so $P_{j}$ can enable transition $\tau_{C}$; then it should leap to $t_{2}$ and so on.

Based on these observations, in the new EEDM, we use one global count-down timer for each system process, e.g., timer $_{i}$ for $P_{i}$ in Figure 4 is set to $\xi_{1}$ on $t_{0}$ and to $\xi_{2}-\xi_{1}$ on $t_{2}$. The Tick process increments now by the value of the smallest timer on condition that no timer equals zero and at least one timer is non-INFINITY. In fact, the Tick process, leaping in this way, is running in the leaping mode; the Tick process in leaping mode and the corresponding system process $P_{i}$ in DVE are described in Figure 5 and Figure $6(N$ is the number of system processes).

\subsubsection{To Know the Current Time Instant}

Careful readers may notice that there is one penalty for Tick to leap: the actual time instant when $\tau_{B}$ is executed is unknown unless it is at $t_{4}$. In fact, in the leaping mode, it is only known that a transition is 

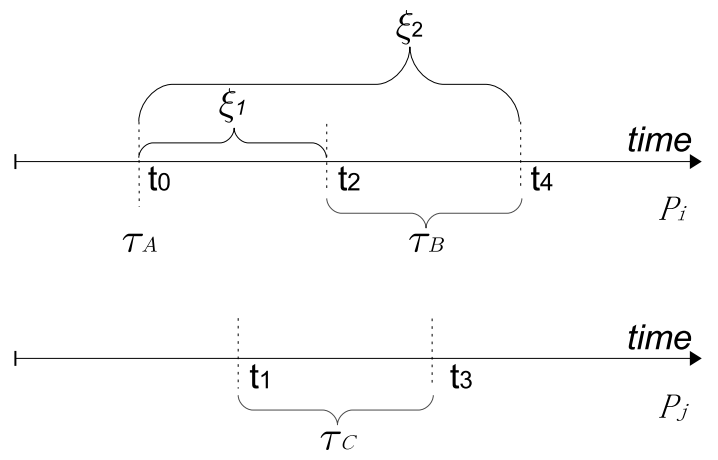

Figure 4: Timeline of process $P_{i}$ and $P_{j}$

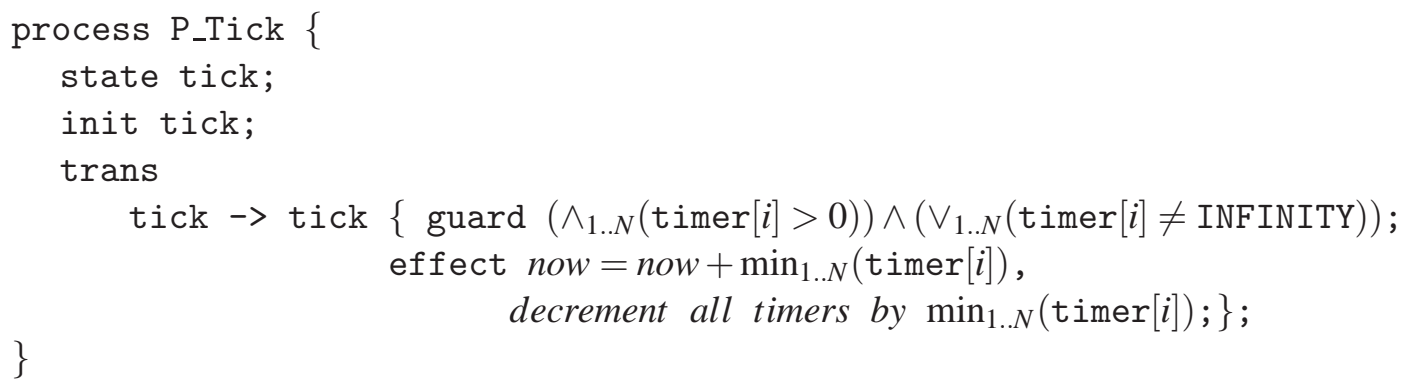

Figure 5: Tick process in leaping mode in DVE for EEDM

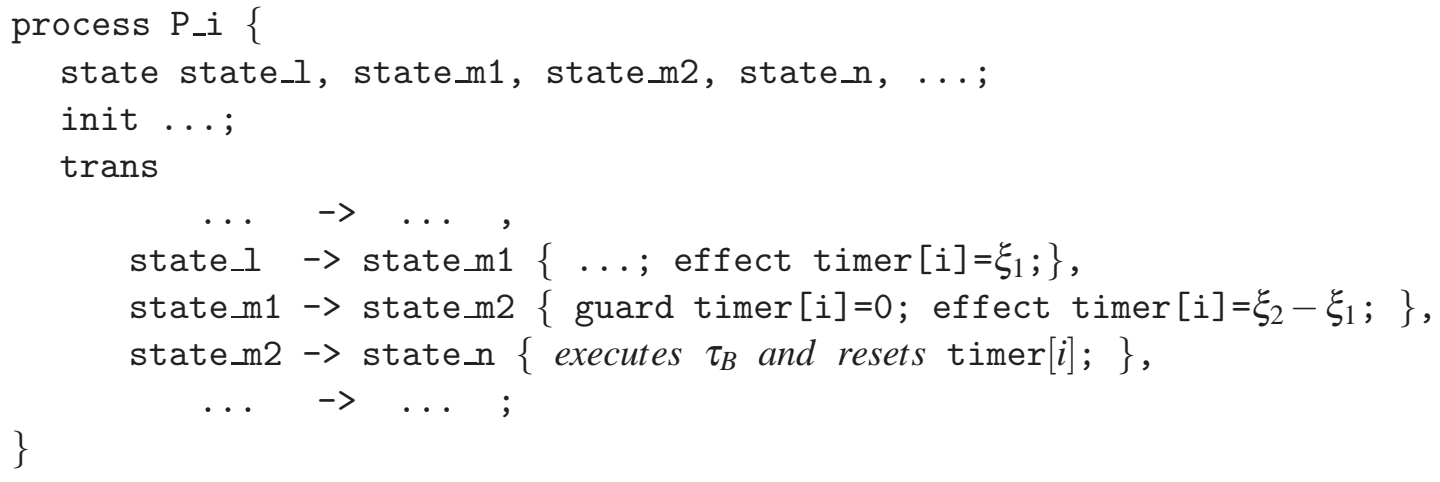

Figure 6: System process $P_{i}$ in DVE for EEDM

executed between the two closest ticks that nest the transition. Consider the example in Figure 4, the Tick will sequentially leap from $t_{0}$ through $t_{4} ; \tau_{B}$ may be executed on: (1) some time instant between $t_{2}$ and $t_{3}$; or (2) some time instant between $t_{3}$ and $t_{4}$; or (3) the time instant of $t_{4}$. However, as we discussed earlier in Section 1 and in [26], in many systems, especially those with pre-emptive scheduling, it is necessary to know the actual time instant when the transition is executed.

To overcome this problem, we allow the Tick process to run in the standard mode. We define a global signal variable for each system process. All signals are set to 0 at the initial state. Whenever a system process $P_{i}$ requires the current time for future calculation, signal $l_{i}$ should be set to 1 ; the Tick process 


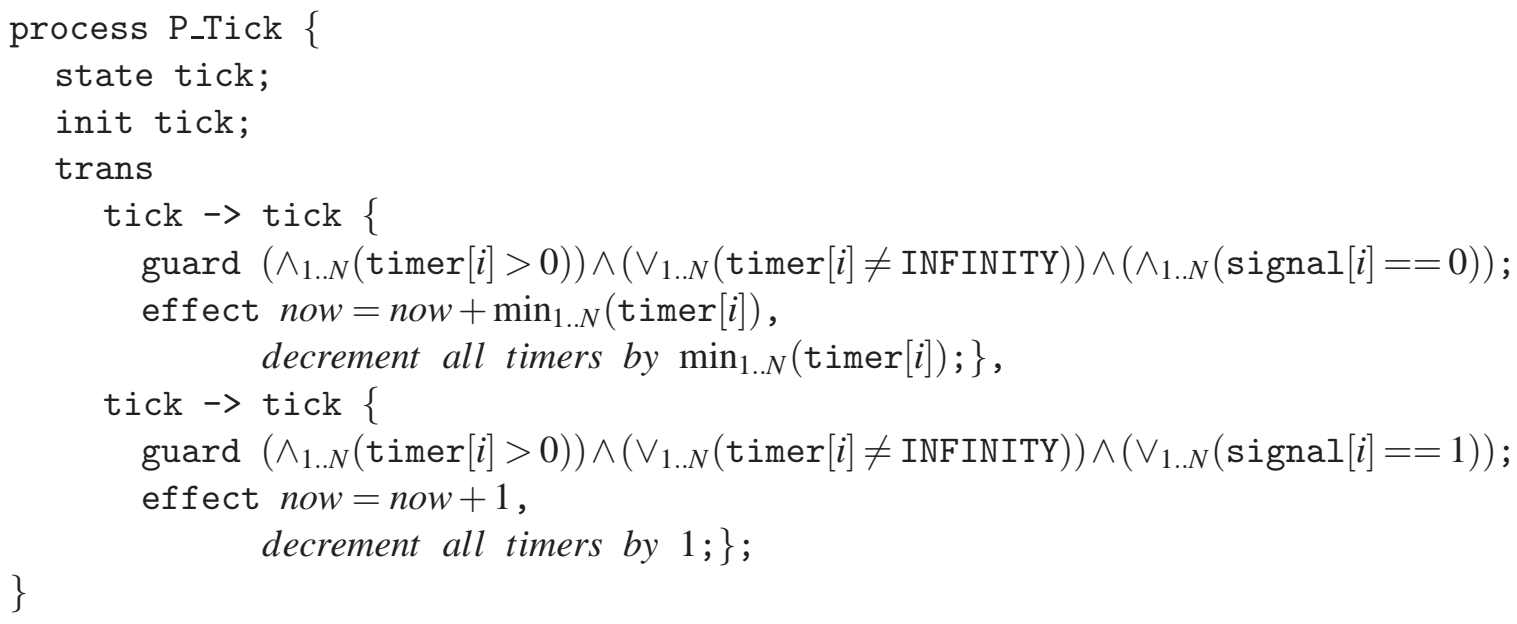

Figure 7: Tick process in standard mode in DVE for EEDM

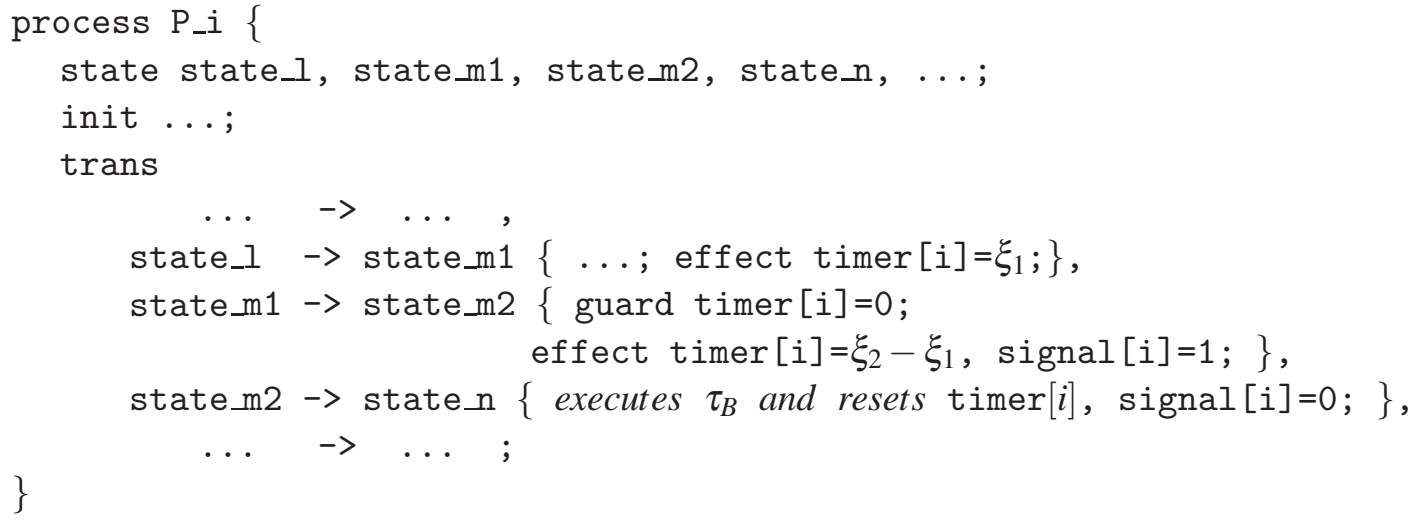

Figure 8: System process $P_{i}$ to illustrate the standard mode

in turn will run in the standard mode with which it will increment now by 1 in each tick. E.g., when time reaches $t_{2}$ in Figure 4, $P_{i}$ 's signal signal ${ }_{i}$ is set to 1 in order to store the time instant at which $\tau_{B}$ is executed; when time reaches $t_{4}$, signal ${ }_{i}$ is set back to 0 so that the Tick switches back to leaping mode. Both the Tick process and the system process need to be updated to incorporate the standard mode, see Figure 7 and Figure 8

\subsubsection{Issues on EDMs and EEDM}

Readers may be concerned about the verification capability of explicit-time description methods. As in our earlier discussion, EDMs simulate a discrete timer by making use of existing constructs in standard un-timed model checkers; in other words, time is just another normal variable in an un-timed model. Therefore, EDMs are not affected by verification issues such as whether the property is specified as an LTL or CTL formula or whether the property is verified using explicit-state based (e.g., Spin) or symbolic model checking (e.g., SMV) algorithms. These verification issues depend on what standard un-timed model checker is used.

Discrete timed model checkers suffer from a common problem: how to find the right time quantum 
(granularity) that does not mask errors. E.g., for processes in a hospital, a time unit defined as a day will definitely mask an error which violates the property "the patient receives a certain treatment within 1 hour". On the other hand, the state space can easily blow up if a finer time unit is used. Readers may be concerned that the introduction of leaping ticks may add to this problem. Actually, leaping ticks do not musk errors in this aspect. The difference between LEDM and EEDM in leaping mode is that EEDM in leaping mode cannot record and use the exact time instant when a transition is executed in the model or the specified properties. For example, the LTL property that $b$ becomes true before 10 time units have elapsed since $\tau_{B}$ is executed cannot be verified using EEDM in leaping mode. For this reason, we introduce the mode-switching mechanism in EEDM.

To reduce the state space, Lamport [17] proposed the use of view symmetry, which is equivalent to abstraction for a symmetric specification $S$. Abstraction consists of checking $S$ by model checking a different specification $A$ called an abstraction of $S$. This technique has two restrictions: (1) the now variable must be eliminated, which means the current time instant is not accessible in this case; (2) if the model checker does not support checking under view symmetry or abstraction, the abstraction specification $A$ must be constructed by hand. In addition, this reduction technique is orthogonal to our EEDM, i.e., we can use Lamport's abstraction technique in conjunction with EEDM.

The idea of leaping ticks in EEDM is quite similar to the notion of time regions in time-automatabased model checkers, which advances time up to the point where a transition must be executed in order not to violate the invariant defined on the corresponding state. However, the implementations are fundamentally different: time-automata-based model checkers introduce specialized data structures [16] to store time regions and use symbolic model checking algorithms extended for time; on the other hand, EEDM, as with LEDM, only uses an explicit tick process and some global variables, and the leaping way of advancing time is obtained by letting the tick leap to the next closest time bound of all systems processes.

\subsubsection{To Know The Current Time Instant: A Pre-emptive Scheduling Example}

Following the triage example described in Section 1 we consider a system of multiple parallel tasks with different priorities, assuming that the right to an exclusive resource is deprivable, i.e., a higher priority task $B$ may deprive the resource from the currently running task $A$. In this case, the elapsed time of $A$ 's execution must be stored for a future resumed execution.

Figure 9 shows a portion of a state transition diagram for task $A$, assuming $A$ needs the exclusive resource $R$ for 10 time units; when $R$ becomes available at time instant $t_{0}, A$ starts its execution by entering the state Exec; at time instant $t_{1}, B$ deprives $A$ 's right to $R$, and $A$ changes to the state Deprived and stores the elapsed $t_{1}-t_{0}$ time units; when $R$ becomes available again, $A$ resumes it execution to state Exec for the remaining $10-\left(t_{1}-t_{0}\right)$ units. Implementation of this example using any one of the three explicit-time description methods is straightforward. Figure 10 shows the process for task $A$ in DVE using EEDM (assuming $A$ has the lowest priority).

\section{Experiments}

\subsection{Overview}

For the convenience of comparison with LEDM in DiVinE, we use the Fischer's mutual exclusion algorithm as in [27] [26]; this algorithm is a well-known benchmark for timed model checking. The description of the algorithm below is adapted from [17]. Our experiment is to model the algorithm in 

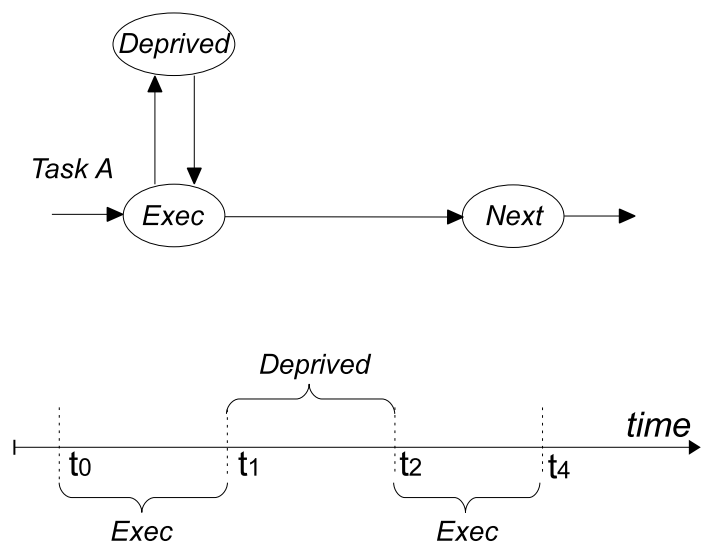

Figure 9: An Example Case of Pre-emptive Scheduling

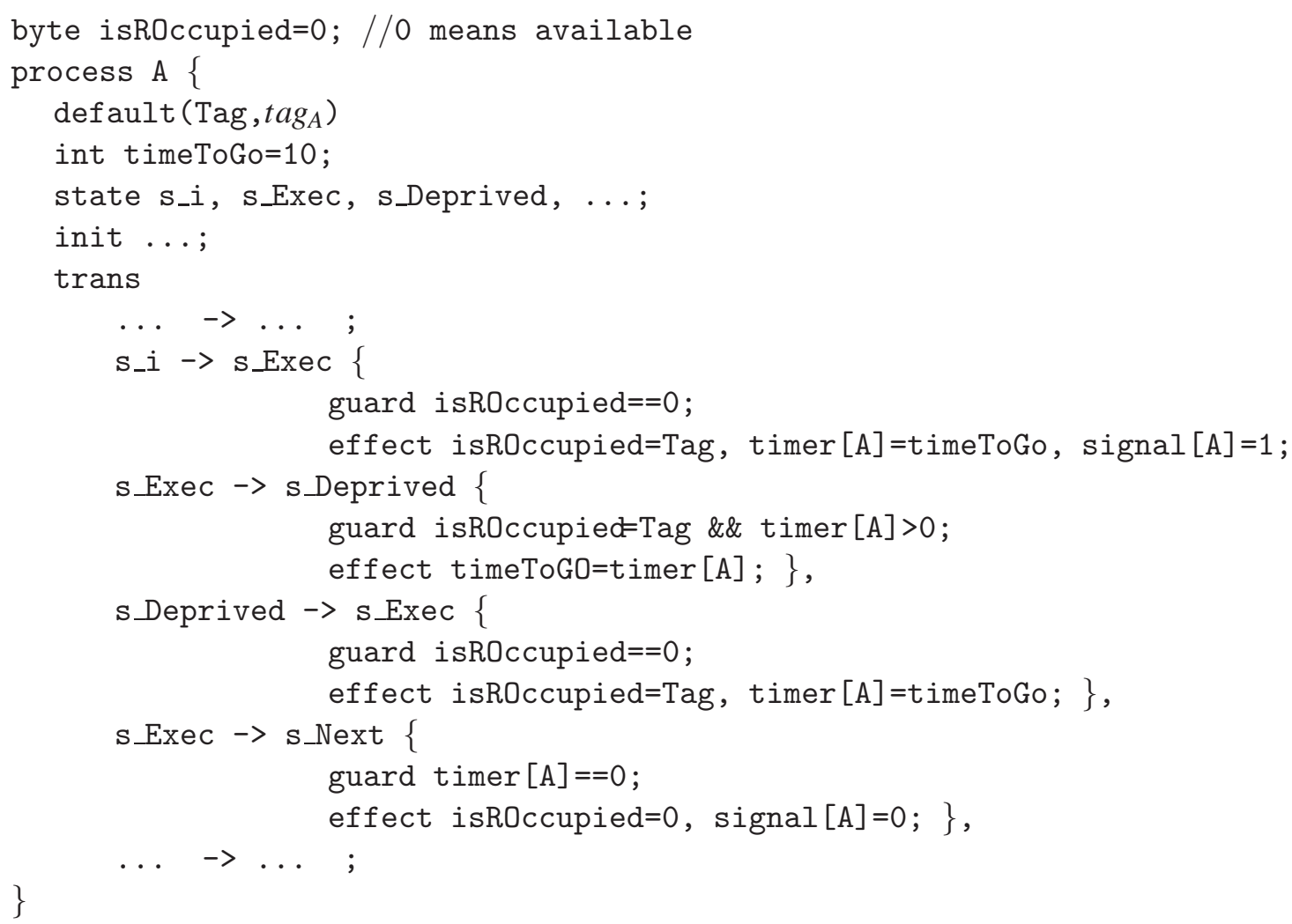

Figure 10: Process in DVE for Pre-emptive Scheduling Example using EEDM

DIVINE using EEDM in both standard and leaping modes, and compare the time and memory efficiency and size of state space with that of LEDM (we omit the experiments for SEDM and SMEDM because they are comparable with LEDM in the aforementioned three numeric criteria). 
Fischer's algorithm is a shared-memory, multi-threaded algorithm. It uses a shared variable $x$ whose value is either a thread identifier (starting from 1) or zero; its initial value is zero. For the convenience of specification of the safety property in our experiments, we use a counter $c$ to count the number of threads that are in the critical section. The program for thread $t$ is described in Figure 11.

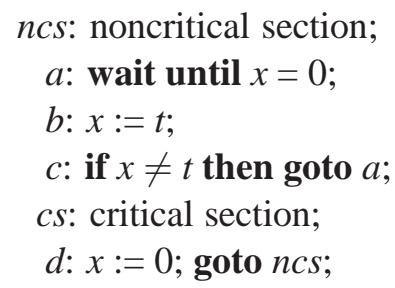

Figure 11: Program of thread $t$ in Fischer's algorithm

The timing constraints are: first, step $b$ must be executed at most $\delta_{b}^{u}$ time units (as an upper bound) after the preceding execution of step $a$; second, step $c$ cannot be executed until at least $\delta_{c}^{l}$ time units (as a lower bound) after the preceding execution of step $b$. For step $c$, there is an additional upper bound $\delta_{c}^{u}$ to ensure fairness, i.e., step $c$ will eventually be executed. The algorithm is tested for 6 threads. The safety property to be verified, "no more than one process can be in the critical section", is specified as $G(c<2)$ for the model.

Version 0.8.1 of the DIVINE-Cluster is used. This version has the new feature of pre-compiling the model in DVE into dynamically linked C functions; this feature speeds up the state space generation significantly. As the example property is known to hold, the OWCTY algorithm is chosen for better time efficiency.

All experiments are executed on the Mahone cluster of ACEnet [1], the high performance computing consortium for universities in Atlantic Canada. The cluster is a Parallel Sun x4100 AMD Opteron (dualcore) cluster equipped with Myri-10G interconnection. Parallel jobs are assigned using the Open MPI library.

\subsection{Experiment 1}

For the first experiment, we use the same value for three constraints, i.e., $\delta_{b}^{u}=\delta_{c}^{l}=\delta_{c}^{u}=T$. Figure 12 compares time and memory efficiency for the two explicit-time description methods with 16 CPUs.

We can see the significant advantage of EEDM in leaping mode: the number of states, verification time and memory usage remain virtually the same for all $T \mathrm{~s}$. Remark that all timing bounds are the same for all threads; the Tick process always leaps $T$ time units in each tick (it ticks only when there is at least one active timer). Therefore, changing the value of $T$ will not change the number of states.

Now we compare LEDM and EEDM in standard mode. Let states $(X)$ be the number of states of method $X$. We can see that, after $T=3$, states $\left(\right.$ EEDM $\left._{\text {standard }}\right)>$ states(LEDM). As $T$ increases from 2 to 9 , states $\left(E E D M_{\text {standard }}\right)$ increases by a factor of 564.9 while states (LEDM) increases by a factor of only 82.2; a comparison of the verification time yields similar results. The system process in EEDM has more transitions than LEDM because there is only one timer for each system process and a timer needs to be assigned twice if the next transition has both lower and upper bounds (e.g. $\tau_{B}$ of $P_{i}$ in Figure 4. timer [i] is assigned to be $\xi_{1}$ and $\xi_{2}-\xi_{1}$ at $t_{0}$ and $t_{2}$ respectively); on the other hand, LEDM has two timers for each system process so assigning both bounds can be made in one step. 


\begin{tabular}{|r|r|r|r|r|r|r|r|r|r|}
\hline & \multicolumn{3}{|c|}{ LEDM } & \multicolumn{5}{|c|}{ EEDM } \\
\cline { 3 - 9 } & \multicolumn{3}{|c|}{$T$} & \multicolumn{3}{|c|}{ standard } & \multicolumn{3}{|c|}{ leaping } \\
\cline { 2 - 9 } & States & Time & Memory & States & Time & Memory & States & Time & Memory \\
\hline 2 & 644,987 & 1.8 & $4,700.1$ & 626,312 & 1.9 & $4,689.6$ & 141,695 & 1.4 & $4,606.2$ \\
3 & $1,438,204$ & 2.4 & $4,822.3$ & $2,375,451$ & 3.4 & $4,982.7$ & 141,695 & 1.5 & $4,612.8$ \\
4 & $3,048,515$ & 3.3 & $4,942.8$ & $7,363,766$ & 5.0 & $5,820.9$ & 141,695 & 1.5 & $4,603.6$ \\
5 & $6,033,980$ & 4.2 & $5,603.4$ & $19,471,191$ & 10.4 & $7,855.2$ & 141,695 & 1.4 & $4,604.9$ \\
6 & $11,201,179$ & 7.2 & $6,343.4$ & $45,552,076$ & 24.4 & $12,241.1$ & 141,695 & 1.4 & $4,620.6$ \\
7 & $19,671,092$ & 11.1 & $7,885.7$ & $96,871,373$ & 52.1 & $20,663.7$ & 141,695 & 1.6 & $4,605.7$ \\
8 & $32,952,899$ & 18.6 & $9,958.9$ & $190,941,594$ & 133.0 & $37,503.6$ & 141,695 & 1.4 & $4,601.8$ \\
9 & $53,025,700$ & 30.2 & $13,288.7$ & $353,811,115$ & 246.5 & $63,572.8$ & 141,695 & 1.4 & $4,622.4$ \\
\hline
\end{tabular}

Figure 12: Number of states, Time (in seconds) and memory usage (in MB) for Experiment 1

\subsection{Experiment 2}

For the second experiment, we set $\delta_{b}^{u}$ and $\delta_{c}^{l}$ to 4 and vary $\delta_{c}^{u}$. Figure 13 compares the number of states, time and memory efficiency for the two explicit-time description methods with 16 CPUs. Figure 14 shows how the size of the state space and verification time grow as $\delta_{c}^{u}$ increases. The extra experimental data for $\delta_{c}^{u}=\{13,14,15,16\}$ are intended to articulate the growing pattern of the state space of EEDM in leaping mode.

\begin{tabular}{|r|r|r|r|r|r|r|r|r|r|}
\hline \multirow{2}{*}{$\delta_{c}^{u}$} & \multicolumn{3}{|c|}{ LEDM } & \multicolumn{4}{|c|}{ EEDM } \\
\cline { 2 - 9 } & \multicolumn{3}{|c|}{ States } & Time & Memory & \multicolumn{3}{c|}{ Standard } & \multicolumn{3}{c|}{ Leaping } \\
\cline { 2 - 9 } & $3,659,317$ & 3.5 & $5,199.1$ & $10,865,877$ & 7.2 & $6,415.6$ & $1,122,491$ & 2.2 & $4,771.0$ \\
6 & $6,783,455$ & 4.2 & $5,770.2$ & $15,221,140$ & 10.2 & $7,150.3$ & $1,046,759$ & 2.0 & $4,758.0$ \\
7 & $12,907,369$ & 7.2 & $6,754.2$ & $21,451,024$ & 13.2 & $8,198.5$ & $3,516,193$ & 3.6 & $5,182.7$ \\
8 & $25,723,697$ & 13.3 & $8,898.8$ & $31,934,332$ & 20.2 & $9,946.8$ & 365,279 & 1.6 & $4,651.1$ \\
9 & $50,500,739$ & 28.2 & $13,047.6$ & $48,889,270$ & 31.2 & $12,721.1$ & $10,998,335$ & 7.1 & $6,434.9$ \\
10 & $93,349,553$ & 52.3 & $20,146.1$ & $73,501,090$ & 50.7 & $16,858.4$ & $3,828,687$ & 3.8 & $5,228.0$ \\
11 & $161,886,059$ & 111.9 & $31,722.6$ & $108,005,926$ & 78.5 & $23,104.9$ & $46,149,106$ & 24.9 & $12,313.8$ \\
12 & $266,256,377$ & 199.2 & $49,154.8$ & $154,662,946$ & 112.2 & $30,045.6$ & 857,773 & 1.9 & $4,735.3$ \\
13 & & & & & & & $92,147,198$ & 48.4 & $19,928.2$ \\
14 & & & & & & & $12,275,835$ & 7.3 & $6,650.4$ \\
15 & & & & & & & $180,459,742$ & 114.1 & $34,098.7$ \\
16 & & & & & & & $1,847,395$ & 2.7 & $4,911.5$ \\
\hline
\end{tabular}

Figure 13: Number of states, Time (in seconds) and memory usage (in MB) for Experiment 2

As opposed to the results in experiment 1, in this experiment EEDM in standard mode performs better than LEDM. We can see that after $\delta_{c}^{u}=9$, states $\left(\right.$ EEDM $\left._{\text {standard }}\right)<\operatorname{states}($ LEDM); as the model becomes larger, states $\left(\mathrm{EEDM}_{\text {standard }}\right)$ increases more slowly than states(LEDM). In fact, as $\delta_{c}^{u}$ increases from 5 to 12 , states(LEDM) increases by a factor of 72.8 while states(EEDM standard $_{\text {increases }}$ by a factor of only 14.2 ; we can see similar comparison results in terms of the verification time. 

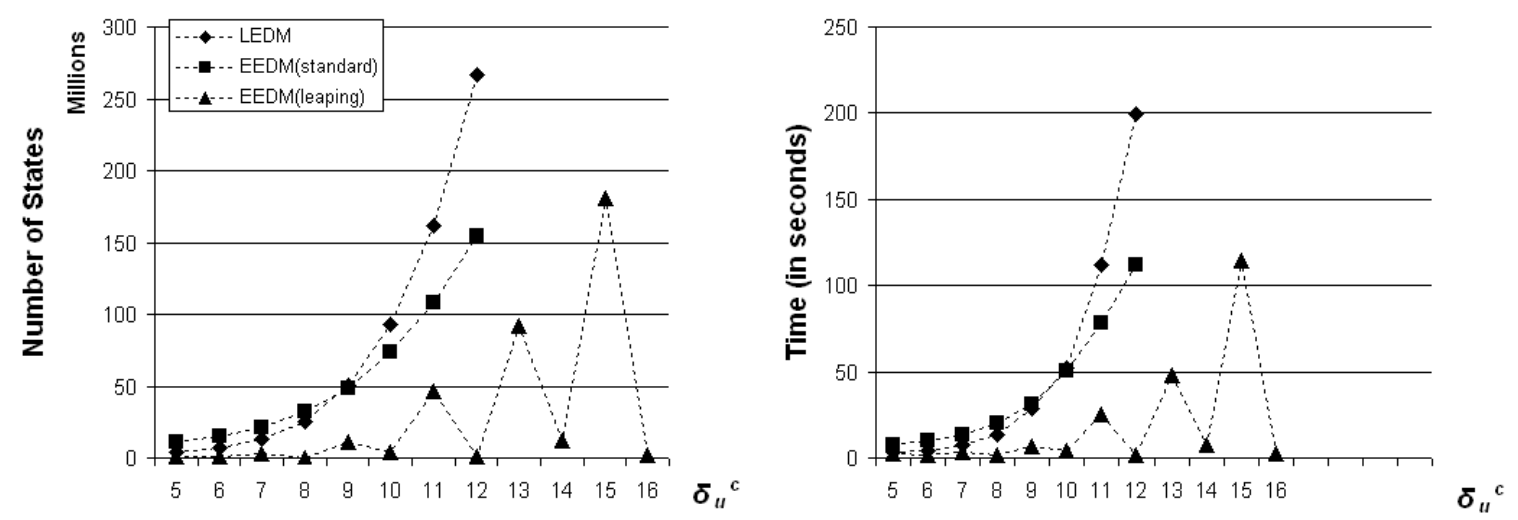

Figure 14: Number of states and Time (in seconds) for Experiment 2

EEDM in leaping mode still shows much better performance than LEDM and EEDM in standard mode; states $\left(\mathrm{EEDM}_{\text {leaping }}\right)$ also shows an interesting phenomenon as $\delta_{c}^{u}$ increases. The number of states of both EEDM in standard mode and LEDM increase at a relatively more steady speed: as $\delta_{c}^{u}$ in-

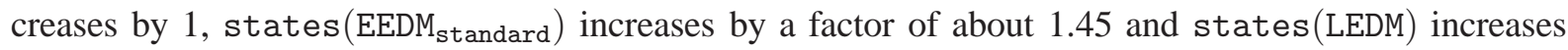
by a factor of about 1.8. On the other hand, the increments of states $\left(\mathrm{EEDM}_{\text {leaping }}\right)$ are grouped by the value of $s=\left(\delta_{c}^{u} \bmod \delta_{c}^{l}\right)$. We can see that, for the same $\left\lfloor\frac{\delta_{c}^{u}}{\delta_{c}^{l}}\right\rfloor$, states $\left(\mathrm{EEDM}_{\text {leaping }}\right)_{\mathrm{s}=0}<$ states $\left(\mathrm{EEDM}_{\text {leaping }}\right)_{\mathrm{s}=2}<$ states $\left(\mathrm{EEDM}_{\text {leaping }}\right)_{\mathrm{s}=1}<\operatorname{states}\left(\mathrm{EEDM}_{\text {leaping }}\right)_{\mathbf{s}=3}$. For $s=0$, whenever there is more than one active timer, their values are integer multiples of $\delta_{c}^{l}$ ( 4 in this experiment), so the Tick still leaps at least 4 time units each tick; in the case of $s=2$, the Tick leaps at least 2 time units each tick. On the other hand, for $s=1$ and $s=3$, in the worst case, the Tick leaps only 1 time units each tick. From these observations, we can conclude that EEDM in leaping mode performs better the greater the greatest common divisor ( $\mathrm{gcd}$ ) of all timing bounds of all system processes.

\section{Conclusion}

In this paper, we present a new explicit-time description method, Efficient Explicit-time Description Method (EEDM) which is significantly more efficient than LEDM, SEDM and SMEDM. In addition to the improved efficiency, EEDM still retains the ability to store and access the current time for future calculations in the system model. Altogether, we have devised methods that have advantages in different aspects of real-time modeling: SEDM and SMEDM have better modularity and adaptability; EEDM is more efficient. These explicit-time description methods provide systematic ways to represent discrete time in un-timed model checkers like SPIN, SMV and DiVINE.

In fact, the explicit-time description methods are intended to offer more options for the verification of real-time systems. First, as Van den Berg et al. mention in [9], in some real-world scenarios when significant resources have been invested into the model for a standard model checker, it is much easier and therefore preferable to extend the existing model to represent time notions rather than re-modeling the entire system for a specialized timed model checker. Second, explicit-time description methods provide a solution for accessing and storing the current clock value for timed-automata-based model checkers. Last and most important, explicit-time description methods, especially the EEDM, enable the usage of 
large-scale distributed model checkers so that we can verify much bigger real-time systems.

This research is part of an ambitious research and development project, Building Decision-support through Dynamic Workflow Systems for Health Care [21]. Real world workflow processes can be highly dynamic and complex in a health care setting. Verification that the system meets its specifications is essential. Standard workflow patterns are widely used in business processes modeling, so we have translated most of the control-flow patterns into DVE and applied them in verifying two small process models [18]. As a continuous effort, we will incorporate explicit-time description methods into workflow patterns' DVE specification and verify a larger model of the real-world healthcare processes with timing information.

As a more complex case study of EEDM, we are now building a pre-emptive scheduling model in the setting of the Dynamic Voltage Scaling (DVS) technique. We also plan to study the possibility of applying different abstraction techniques to the explicit-time description methods: Dutertre and Sorea [13] and Clarke et al. [11] recently presented two different abstraction techniques for timed automata and the abstraction outcome can be verified using un-timed model checkers.

\section{Acknowledgment}

This research is sponsored by Natural Sciences and Engineering Research Council of Canada (NSERC), an Atlantic Computational Excellence Network (ACEnet) Post Doctoral Research Fellowship and the Atlantic Canada Opportunities Agency (ACOA) through an Atlantic Innovation Fund project. The computational facilities are provided by ACEnet. We thank Jiri Barnat, Keith Miller and the anonymous reviewers of PDMC'09 for their valuable comments.

\section{References}

[1] Atlantic Computational Excellence network (ACEnet). http://www.ace-net.ca/. Last accessed on Nov. 2009.

[2] DiVinE project. http://divine.fi.muni.cz/. Last accessed on Nov. 2009.

[3] Yasmina Abdeddaïm \& Oded Maler (2002): Preemptive Job-Shop Scheduling Using Stopwatch Automata. In: Joost-Pieter Katoen \& Perdita Stevens, editors: TACAS, Lecture Notes in Computer Science 2280. SpringerVerlag, pp. 113-126.

[4] Rajeev Alur \& David L. Dill (1994): A Theory of Timed Automata. Theor. Comput. Sci. 126(2), pp. 183-235.

[5] Rajeev Alur \& Thomas A. Henzinger (1991): Logics and Models of Real Time: A Survey. In: J. W. de Bakker, Cornelis Huizing, Willem P. de Roever \& Grzegorz Rozenberg, editors: REX Workshop, Lecture Notes in Computer Science 600. Springer-Verlag, pp. 74-106.

[6] Jiri Barnat, Lubos Brim \& Ivana Cerná (2005): Cluster-Based LTL Model Checking of Large Systems. In: Frank S. de Boer, Marcello M. Bonsangue, Susanne Graf \& Willem P. de Roever, editors: FMCO, Lecture Notes in Computer Science 4111. Springer-Verlag, pp. 259-279.

[7] Johan Bengtsson, Kim Guldstrand Larsen, Fredrik Larsson, Paul Pettersson \& Wang Yi (1995): UPPAAL - a Tool Suite for Automatic Verification of Real-Time Systems. In: Rajeev Alur, Thomas A. Henzinger \& Eduardo D. Sontag, editors: Hybrid Systems, Lecture Notes in Computer Science 1066. Springer-Verlag, pp. 232-243.

[8] Johan Bengtsson \& Wang Yi (2003): Timed Automata: Semantics, Algorithms and Tools. In: Jörg Desel, Wolfgang Reisig \& Grzegorz Rozenberg, editors: Lectures on Concurrency and Petri Nets, Lecture Notes in Computer Science 3098. Springer-Verlag, pp. 87-124. 
[9] Lionel van den Berg, Paul A. Strooper \& Kirsten Winter (2007): Introducing Time in an Industrial Application of Model-Checking. In: Stefan Leue \& Pedro Merino, editors: FMICS, Lecture Notes in Computer Science 4916. Springer-Verlag, pp. 56-67.

[10] Lubos Brim (2004): Parallel Model Checking. ERCIM News 2004(58), pp. 35-36.

[11] Edmund M. Clarke, Flavio Lerda \& Muralidhar Talupur (2007): An Abstraction Technique for Real-time Verification. In: S. Ramesh \& P. Sampath, editors: Next Generation Desigh and Verification Methodologies for Distributed Embedded Control Systems: Proceedings of the General Motors Research and Development Workshop, Lecture Notes in Computer Science. Springer-Verlag, pp. 1-17.

[12] David L. Dill (1989): Timing Assumptions and Verification of Finite-State Concurrent Systems. In: Joseph Sifakis, editor: Automatic Verification Methods for Finite State Systems, Lecture Notes in Computer Science 407. Springer-Verlag, pp. 197-212.

[13] Bruno Dutertre \& Maria Sorea (2004): Modeling and Verification of a Fault-Tolerant Real-Time Startup Protocol Using Calendar Automata. In: Yassine Lakhnech \& Sergio Yovine, editors: FORMATS/FTRTFT, Lecture Notes in Computer Science 3253. Springer-Verlag, pp. 199-214.

[14] Thomas A. Henzinger, Zohar Manna \& Amir Pnueli (1992): What Good Are Digital Clocks? In: Werner Kuich, editor: ICALP, Lecture Notes in Computer Science 623. Springer-Verlag, pp. 545-558.

[15] Gerard J. Holzmann (1991): Design and Validation of Computer Protocols. Prentice Hall.

[16] Pavel Krcál \& Wang Yi (2004): Decidable and Undecidable Problems in Schedulability Analysis Using Timed Automata. In: Kurt Jensen \& Andreas Podelski, editors: TACAS, Lecture Notes in Computer Science 2988. Springer-Verlag, pp. 236-250.

[17] Leslie Lamport (2005): Real-Time Model Checking Is Really Simple. In: Dominique Borrione \& Wolfgang J. Paul, editors: CHARME, Lecture Notes in Computer Science 3725. Springer-Verlag, pp. 162-175.

[18] Nazia Leyla, Ahmed Mashiyat, Hao Wang \& Wendy MacCaull (2009): Workflow Verification with DiVinE. In: Parallel and Distributed Methods in verifiCation, 8th International Workshop, PDMC 2009, Held as Part of the Formal Methods Week 2009, Eindhoven, the Netherlands, November 2-6, 2009. Short paper.

[19] Ken L. McMillan (1992): Symbolic model checking - an approach to the state explosion problem. Ph.D. thesis, Carnegie Mellon University.

[20] Philip M. Merlin (1974): A study of the recoverability of computing systems. Ph.D. thesis, Department of Information and Computer Science, University of California, Irvine, CA.

[21] Keith Miller \& Wendy MacCaull (2009): Toward Web-based Careflow Management Systems. Journal of Emerging Technologies in Web Intelligence (JETWI) Special Issue, E-health: Towards System Interoperability through Process Integration and Performance Management. Accepted.

[22] Wojciech Penczek \& Agata Pólrola (2004): Specification and Model Checking of Temporal Properties in Time Petri Nets and Timed Automata. In: Jordi Cortadella \& Wolfgang Reisig, editors: ICATPN, Lecture Notes in Computer Science 3099. Springer-Verlag, pp. 37-76.

[23] John H. Reif (1985): Depth-First Search is Inherently Sequential. Inf. Process. Lett. 20(5), pp. 229-234.

[24] Moshe Y. Vardi \& Pierre Wolper (1986): An Automata-Theoretic Approach to Automatic Program Verification (Preliminary Report). In: LICS. IEEE Computer Society, pp. 332-344.

[25] Kees Verstoep, Henri E. Bal, Jiri Barnat \& Lubos Brim (2009): Efficient large-scale model checking. In: IPDPS. IEEE, pp. 1-12.

[26] Hao Wang \& Wendy MacCaull (2009): Timed Model Checking with Explicit-time Description Methods. Technical Report StFX-CLI-TR-2009-03, Centre for Logic and Information, St. Francis Xavier University.

[27] Hao Wang \& Wendy MacCaull (2009): Verifying Real-Time Systems using Explicit-time Description Methods. In: 16th International Symposium on Formal Methods Workshop, Quantitative Formal Methods: Theory and Applications, QFM 2009, Eindhoven, the Netherlands, November 3, 2009.

[28] Sergio Yovine (1997): KRONOS: A Verification Tool for Real-Time Systems. STTT 1(1-2), pp. 123-133. 